\title{
Western diet in ApoE-LDLR double-deficient mouse model of atherosclerosis leads to hepatic steatosis, fibrosis, and tumorigenesis
}

\author{
Marian Kampschulte ${ }^{1}$, Christiane Stöckl ${ }^{1}$, Alexander C Langheinrich ${ }^{2}$, Ulrike Althöhn ${ }^{1}$, Rainer M Bohle ${ }^{3}$, \\ Gabriele A Krombach ${ }^{1}$, Philipp Stieger ${ }^{4}$, Yuri Churin ${ }^{5}$, Sandra Kremer ${ }^{5}$, Christian Dierkes ${ }^{6}$, Timo Rath ${ }^{5,7}$, Elke Roeb ${ }^{5}$ \\ and Martin Roderfeld ${ }^{5}$
}

Nonalcoholic fatty liver disease has been linked to cardiovascular diseases and atherosclerosis. The aim of the current study was to characterize the hepatic pathology leading to fibrosis and tumors in a murine model of atherosclerosis. Male apolipoprotein E/low-density lipoprotein receptor double-knockout mice (AL) mice were fed with a high fat and high cholesterol western diet for 35 weeks (AL mice on WD). Protein and mRNA analysis as well as micro-computed tomography (micro-CT) were performed to assess oxidative stress, liver damage, inflammation, fibrosis, signaling pathways, vascularization, and tumorigenesis. Controls were chosen to distinguish between genetically and dietary effects in steatohepatitis and associated tumorigenesis. Hepatic inflammation and dyslipidemia were increased in AL mice on WD compared with wild-type mice on WD. Uniquely, AL mice on WD showed a spontaneous development of tumors (30\% of cases) and thickening of intrahepatic vessel walls. Functionally relevant underlying signaling pathways such as NF- $\kappa$ B, Stat3, JNK, and AKT were differentially regulated between AL and wild-type mice on WD. Micro-CT was capable of visualizing and quantitatively distinguishing tumor neovascularization from vascularization in non-neoplastic liver tissue. AL mice on WD diet represent a novel model combining atherosclerosis and nonalcoholic fatty liver disease. Signaling pathways of liver cell damage and compensatory liver regeneration in combination with enhanced inflammation appear to be crucial for the spontaneous development of tumors in AL mice on WD. Micro-CT represents a new and powerful technique for the ultrastructural and three-dimensional assessment of the vascular architecture of liver tumors.

Laboratory Investigation (2014) 94, 1273-1282; doi:10.1038/labinvest.2014.112; published online 8 September 2014

Nonalcoholic fatty liver disease (NAFLD) is an emerging important public health problem across the globe and is associated with high risk for cardiovascular disease in adults and children. ${ }^{1}$ NAFLD encompasses a continuum of diseases, including simple steatosis and non-alcoholic steatohepatitis (NASH) as a necroinflammatory disease of the liver. NAFLD and NASH became epidemic in western countries. Depending on the screening tool and when the study was performed, the estimated prevalence of NAFLD ranges from 2.8 to $46 \% .^{2,3}$ Importantly up to $25 \%$ of NAFLD patients develop NASH, and in approximately one-third of NASH patients there is slowly progressive liver fibrosis leading to cirrhosis. $^{4,5}$ In 1998, the 'two hit' theory of disease pathogenesis was proposed in which lipid accumulation and steatosis as first hit sensitizes the liver to a variety of second hits (eg, oxidative stress) that lead to necroinflammation and fibrosis. ${ }^{6}$ Recent experimental data suggest, however, that dietary cholesterol intake rather than existing liver steatosis represents a major risk for the development of NASH. ${ }^{7}$ Dyslipidemia is, apart from obesity and diabetes mellitus type II, another important risk factor for the development of NAFLD. ${ }^{8}$

To improve our understanding of pathomechanisms underlying NAFLD-associated vascular diseases, animal models resembling both pathologies are urgently needed. These models require the existence of defined criteria for

\footnotetext{
'Department of Radiology, Justus-Liebig University Giessen, Giessen, Germany; ${ }^{2}$ Department of Diagnostic and Interventional Radiology, BG Trauma Hospital, Frankfurt/Main, Germany; ${ }^{3}$ Department of Pathology, Saarland University, Homburg, Germany; ${ }^{4}$ Department of Cardiology, Angiology and Pneumology, Otto von Guericke University Magdeburg, Magdeburg, Germany; ${ }^{5}$ Department of Gastroenterology, Justus-Liebig University Giessen, Giessen, Germany; ${ }^{6}$ MVZ for Pathology, Trier, Germany and ${ }^{7}$ Department of Medicine 1, Friedrich-Alexander University Erlangen-Nuremberg, Erlangen, Germany Correspondence: Dr M Roderfeld, PhD, Department of Gastroenterology, Justus-Liebig University Giessen, Gaffkystr. 11, Giessen 35392, Germany. E-mail: martin.roderfeld@innere.med.uni-giessen.de 
NAFLD, such as hepatic steatosis, inflammation, fibrosis, liver cell damage, metabolic changes, and risk factors.

$A p o E^{-1-}-L D L R^{-1-}(\mathrm{AL})$ mice are currently established in atherosclerosis research, ${ }^{9}$ and although inflammation in $\mathrm{AL}$ mice has been demonstrated in various organs, little is known about hepatic disease in these mice. The aim of the present study was to provide a detailed characterization of the hepatopathy in AL mice on $\mathrm{C} 57 \mathrm{Bl} / 6$ background on western diet (WD), including analysis of signaling pathways, which were associated with cancerogenesis. ${ }^{10-12}$ Within this new model for both atherosclerosis and NAFLD, we further assessed the value of quantitative micro-CT for vascular imaging within neoplastic liver pathologies.

\section{MATERIALS AND METHODS}

Animal studies were performed according to the 'German Animal-Protection Law' (1993) and approved by the institutional animal care and use committee. Ten AL mice (B6.129-Apoetm1Unc Ldlrtm1Her/J, Jackson Laboratory, Bar Habor, USA) and five wild-type (WT) mice (C57Bl/6) were fed for 35 weeks with a cholesterol (5\%) and fat (21\%) enriched WD (SSNIFF, Soest, Germany \#EF TD88137, composition of diets: Supplementary Table S1). Five AL littermates and six $\mathrm{C} 57 \mathrm{Bl} / 6$ mice of the same age received a regular diet (RD) and served as controls (Supplementary Figure S1). All experiments were performed with male mice. Mice were allocated randomly to experimental groups.

Fresh liver tissue and blood samples were obtained from five WD-fed AL and WT mice and from five standard-fed AL and six C57BL/6 mice with RD. Serum was examined for ALT, AST, AP, triglycerides, and cholesterol levels utilizing the Reflotron-system (Roche, Mannheim, Germany).

\section{qRT-PCR}

mRNA isolation, cDNA synthesis, quantitative real-time PCR, quality control, and data analyses were performed as described before. ${ }^{13}$

\section{Western Blotting}

Total liver lysates were immunoblotted using antibodies against fatty acid synthetase (FASN), $\beta$-actin, phosphoSAPK/JNK, AKT, I $\kappa$ B, phospho-STAT3, STAT3, phosphoAKT (preceding antibodies from Cell Signaling/NEB, Frankfurt, Germany), and NF- $\kappa \mathrm{B} / \mathrm{p} 65$ (BioLegend, Fell, Germany).

\section{Histology}

Specimens were embedded in paraffin, serial sectioned at $6-\mu \mathrm{m}$ slice thickness, and stained with H\&E, Oil Red O, and Masson-Goldner trichrome. Histomorphometric analysis of hepatic grading and staging and steatosis scoring were assessed by a liver pathologist (CD) on semiquantitative scores, as published elsewhere. ${ }^{14-16}$ To assess levels of superoxide anions, freshly prepared frozen sections were stained with dihydroethidine hydrochloride as previously described. ${ }^{17}$

\section{Immunohistological Analysis}

Immunostaining on cryoslices was performed using MMP-9 (R\&D Systems, Wiesbaden, Germany), MMP-13 (Dianova, Hamburg, Germany), Typ-I Collagen (BioMol, Hamburg, Germany), GFAP (Calbiochem, San Diego, USA), CD45 (BD, Heidelberg, Germany), and $\alpha$-SMA (Progen, Heidelberg, Germany) antibodies. Immunofluorescence staining was performed as described before. ${ }^{13}$ Paraformaldehyde-fixed paraffin-slices were stained using type IV collagen, MCM2, PCNA (Cell Signaling/NEB, Frankfurt, Germany), CK-18, and $\alpha$-SMA (CK-18 and SMA from Progen, Heidelberg, Germany) antibodies.

\section{Hydroxyproline Assay}

Liver collagen content was determined by hydroxyproline (HYP) quantification as described before. ${ }^{18}$

\section{MMP Zymography}

Expression of MMP-9 protein was analyzed by gelatin zymography as described before..$^{19} \mathrm{In}$ all, $1 \mathrm{mg} / \mathrm{ml}$ gelatin was copolymerized in a 7.5\% PAGE gel, and $10 \mu \mathrm{g}$ of liver lysate protein was loaded.

\section{Micro-CT Imaging}

A lead containing polymer (Microfil MV-122, Flow Tech, Carver, MA, USA) was injected into the left ventricle of five killed WD-fed AL mice with a nominal pressure of $100 \mathrm{~mm} \mathrm{Hg}$. Livers were removed en bloc after polymerization of the contrast agent and immersed in $4 \%$ neutral buffered formalin. Each lobe of the livers was scanned using a micro-CT system (Micro-CT_1072, SkyScan, Kontich, Belgium) at $14-\mu \mathrm{m}$ voxel size. Samples were scanned over 180 degrees in steps of 0.45 degrees.

3D data sets were examined by two radiologists (ACL; MK) for tumor-suspicious structural abnormalities (vascular branching pattern, vascular proliferation, elongation, and displacement of vessels). Quantitative measurements were performed using the CTAn Software (CTAn 1.12, Bruker MicroCT, Kontich, Belgium). Quantified parameters were tumor volume, vascular volume fraction (VVF\%), and vessel size distribution. Cubic volumes of interest with a size of 4-8 cubic millimeters were used to quantify vascular parameters in non-neoplastic liver tissue and tumor tissue. VVF was calculated as follows: $\mathrm{VVF}=\mathrm{TCAV} / \mathrm{TTV} \times 100 \%$. TCAV equals the total volume of contrast agent in the examined volume of interest; TTV is the total tissue volume.

\section{Statistics}

Statistical analysis was performed with SPSS 19.0 (IBM, New York, USA). For non-normally distributed parameters, non-parametric tests (Mann-Whitney $U$ test, Spearman's rank test) were applied. Data are shown as mean \pm s.e.m. A two-sided $P<0.05$ was considered significant. 


\section{RESULTS}

\section{Lipid Metabolism and Dyslipidemia after Long-Term Nutrition with WD}

We intended to analyze parameters of lipid metabolism and dyslipidemia to assess the effects of a fat- and cholesterolenriched WD. As expected, triglyceride serum levels were significantly enhanced in AL mice on WD and RD compared with WT mice (Figure 1a). Similarly, serum cholesterol was strongly increased in AL mice irrespective of the diet compared with WT mice (Figure 1a). In particular, serum cholesterol levels were increased 7-fold in AL mice on RD and 15 -fold in AL mice on WD diet, indicating that the combination of genotype and WD leads to most profound changes in lipid and cholesterol metabolism.

Consistent with these findings, peroxisome proliferator activated receptor (PPAR) $-\alpha$ and acyl-CoA oxidase (ACO) as key factors of lipid metabolism were increased only in AL mice on WD (Figure $1 b$ ) but not in AL mice on RD or WT mice. PPAR- $\gamma$, however, was induced not only in AL mice and WT on WD but also in AL mice on RD. FASN protein levels were increased in AL mice and WT mice on WD in comparison to WT and AL mice on RD (Figure 1c), indicating that FASN is mainly regulated by the diet itself.

\section{Hepatic Inflammation and Hepatocellular Injury under WD and Genetic Predisposition}

Having shown profound changes in lipid metabolism in AL mice on WD, we then assessed hepatic inflammation and hepatocellular injury in WT and AL mice on RD and WD. Microvesicular and macrovesicular steatosis was readily visible in both WT and AL mice on WD (Figure 2a), as assessed in H\&E stainings. When quantitatively scoring the histopathological grading of inflammation, AL mice on WD exhibited the highest grading scores with a significant increase compared with littermates on RD and WT mice on $\mathrm{WD}$, thereby indicating that the combination of genotype and WD causes the most profound inflammatory phenotype (Figures 2a and b). Consistent with this, the number of infiltrated leukocytes and inflammatory foci was most pronounced and prominent in $\mathrm{AL}$ mice on WD (Figure 2c, Supplementary Figure S5a). As a further corroboration, AL mice on RD exhibited a significantly increased steatosis score compared with WT mice on RD, which was further increased in AL mice on WD (Figure 2b). The expression of the pro-inflammatory cytokines TNF- $\alpha$, IL-12, and IFN- $\gamma$ were increased in both WT and AL mice on WD (Supplementary Table S2). IL-12, however, was also increased in AL mice on RD (Supplementary Table S2).

Supplementary Figure S5b demonstrates increased numbers of hepatic macrophages in AL mice on RD and WT mice on WD in comparison to WT mice on RD. Nevertheless, macrophage infiltration peaked in AL mice on WD, which is consistent with increased infiltration of CD45 ${ }^{+}$leukocytes: Comparable to Figure $2 \mathrm{c}$, nests of infiltrated macrophages appeared in the same manner as $\mathrm{CD} 45^{+}$leukocytes (red arrows in Supplementary Figure S5b).

Enhanced numbers of infiltrated myeloperoxidase ${ }^{+}$ neutrophils and $\mathrm{CD}^{+}$T-cells could be visualized in the liver of WD-treated mice while AL mice on RD showed no difference to WT mice on RD (Supplementary Figures S5c and $\mathrm{d}$ ).

Taken together, these data indicate that the combination of genetic predisposition and high dietary fat causes the most profound hepatic inflammation.

As established markers of liver cell damage which are also seen in humans, AL and WT mice on WD exhibited ballooned hepatocytes, cytoskeletal collapse, and Mallory body formation, thereby confirming liver and hepatocyte damage on cellular level (Figure 2d and Supplementary Figures S2a and $\mathrm{b}$ ). Consistent with this histological assessment of liver cell damage, serum levels of AST, ALT, and AP were increased not only in AL mice but also in WT mice on WD compared with littermate controls on RD (Figure 2e). Further, WD induced the hepatic accumulation of ROS in WT and AL mice (Figure 2f), again with most intense stainings observed in AL mice on WD. a

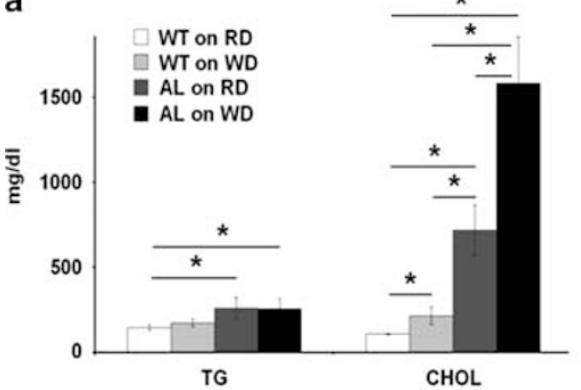

b

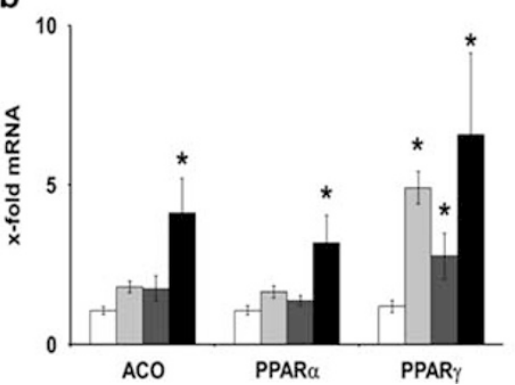

C

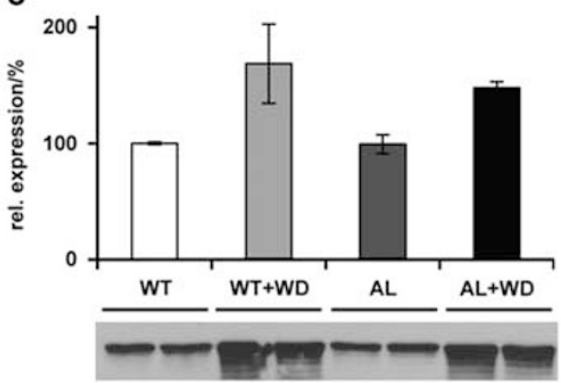

Figure 1 Lipid metabolism and dyslipidemia after long-term nutrition with western diet. (a) Serum triglyceride and cholesterol levels were elevated extraordinary in AL mice on RD and WD. (b) Transcriptional regulation of key factors in fat metabolism. (c) Western blotting analysis revealed enhanced intrahepatic fatty acid synthetase protein expression after WD. Western blotting was quantified by using the BioDocAnalyze software, and mean values were determined. 

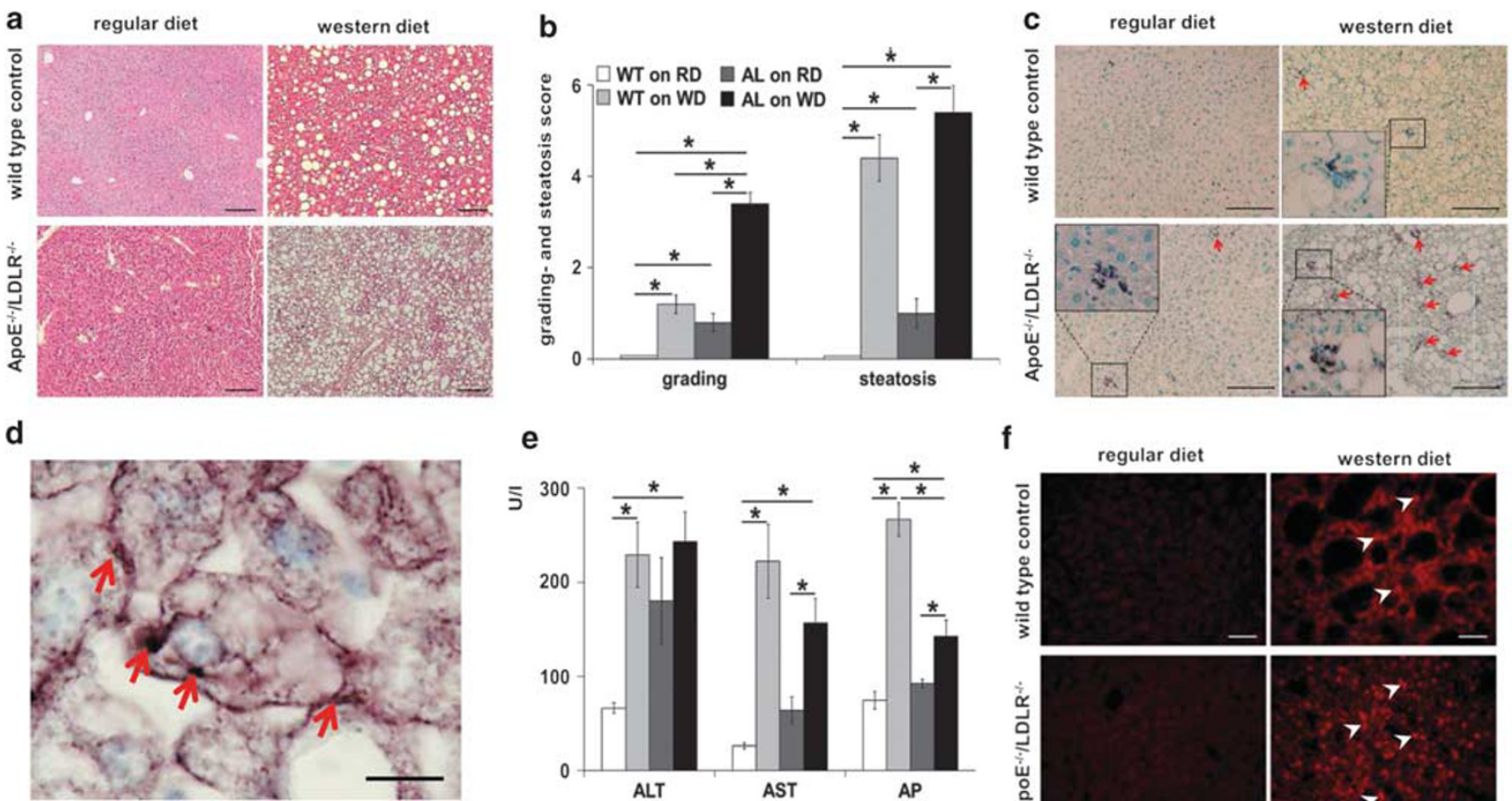

\section{e}

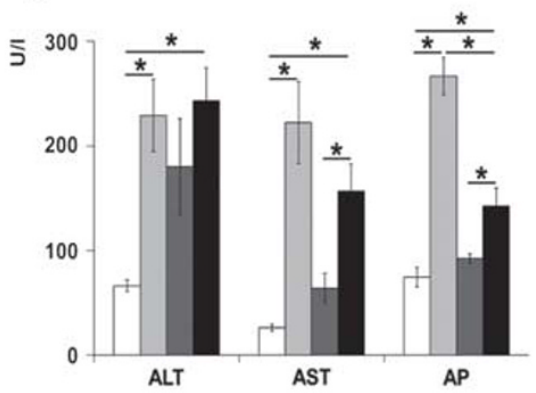

f

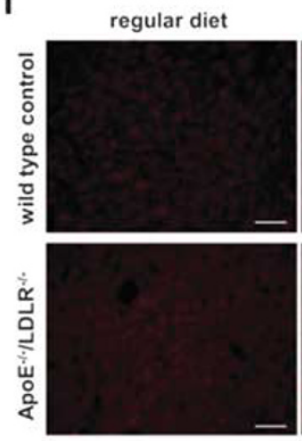

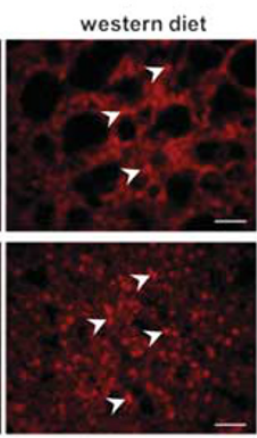

Figure 2 Western diet and ApoE/LDLR double-knockout cause oxidative stress, hepatocellular injury, and inflammation. (a) H\&E staining of WT and AL mice on RD and western diet. Magnification $\times 50$, scale bar $200 \mu \mathrm{m}$. For higher magnification of inflammatory foci, please refer to Supplementary Figures S5 a and b) Inflammatory grading and steatosis scoring were performed by a pathologist, who was blinded to experimental data.

(c) Pan-leukocyte marker CD45 was stained by IHC on 3- $\mu$ m-thick paraffin-embedded liver sections. Red arrows indicate conglomerates of leukocytes. Magnification $\times 100$ and $\times 400$, scale bar $200 \mu \mathrm{m}$. (d) CK-18 immunohistochemistry demonstrated Mallory body formation as a characteristic sign for cytoskeletal breakdown and cellular injury (red arrows) in AL mice after WD. Magnification $\times 1000$, scale bar $10 \mu \mathrm{m}$. (e) ALT, AST, and alkaline phosphatase (AP) increased following WD in WT and AL mice, whereas no elevation was observed in AL littermates on RD. (f) Superoxide accumulation was demonstrated by dihydroethidine hydrochloride (DHE) staining of naive liver slices in mice on WD (arrowheads). Representative micrographs are shown (scale bars $50 \mu \mathrm{m}$ ).

a

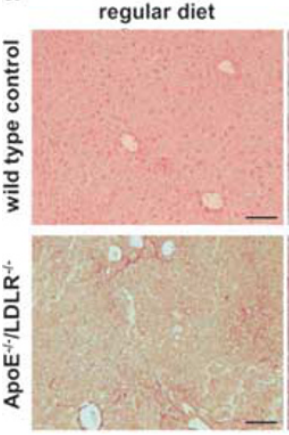

western diet
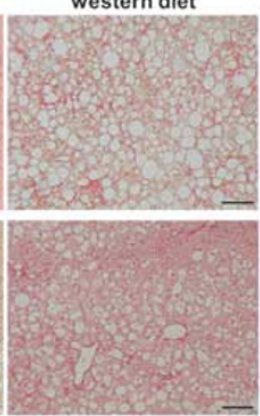

b

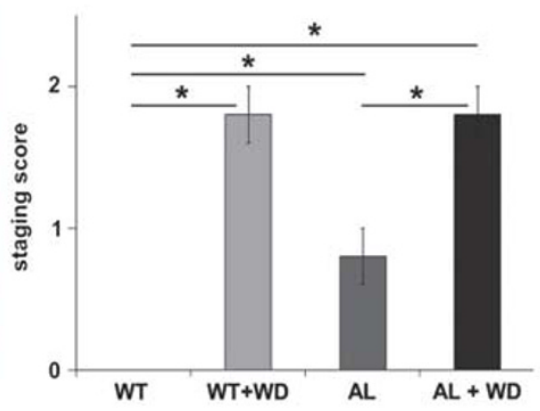

C

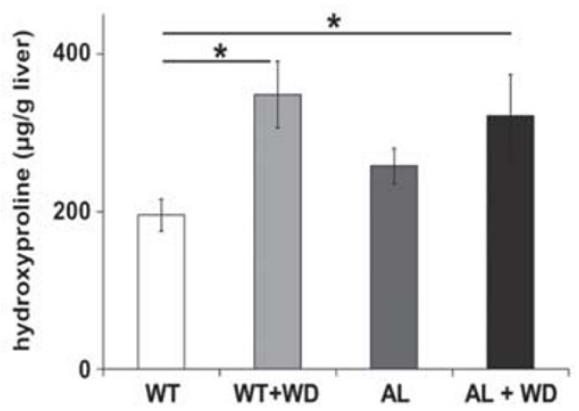

Figure 3 Hepatic fibrosis in mice on western diet. (a) Sirius Red staining was performed on liver paraffin sections. Enhanced chicken wire fibrosis appeared along with sinusoids and periportal fibrillar collagen deposition in WT and AL mice on WD (upper right and lower right panels). Even in AL mice on RD, moderately enhanced chicken wire fibrosis was stained (lower left panel). Faint red staining in WT mice on RD is limited to nuclei. Magnification $\times 100$, scale bar $100 \mu \mathrm{m}$. (b and c) Collagen deposition was quantified by staging and measurement of hepatic hydroxyproline content.

\section{Hepatic Fibrosis in Mice on WD}

Next, we set off to biochemically and histologically quantify hepatic fibrosis. As assessed by staging and hydroxyproline quantification, the amount of hepatic fibrillar collagen was significantly increased in both WT and AL mice on WD
(Figures 3a-c). In Sirius Red and Masson-Goldner stainings, this increased total amount of fibrillar collagen corresponded to periportal and parenchymal chicken wire fibrosis of mild up to pronounced character in AL and WT mice on WD (Figure 3a and Supplementary Figure S2). 
Co-staining for type I collagen and $\alpha$-SMA identified collagen-producing myofibroblasts within these regions of chicken wire fibrosis as a major pro-fibrogenic cell population (Supplementary Figure S3c). Of note, $\alpha$-SMA ${ }^{+}$ myofibroblasts were found to be in close contact to $\mathrm{GFAP}^{+}$ hepatic stellate cells (HSC) but did not co-stain with GFAP (Supplementary Figure S3e). Apart from chicken wire fibrosis, only AL mice on WD regularly exhibited a thickening of intrahepatic vessel walls as a further manifestation of fibrosis, again with the presence of collagen-producing $\alpha$ $\mathrm{SMA}^{+}$myofibroblasts as identified by immunohistochemistry (Supplementary Figures S3a, b, and d). Further, co-staining of elastin and $\alpha$-SMA within portal tracts suggested that portal fibroblasts represent another cellular source for hepatic myofibroblasts in $\mathrm{AL}$ mice on $\mathrm{WD}$ (Supplementary Figure S3f).

Fibrogenic cytokine $T g f-\beta$ was increased in WT and AL mice on WD, whereas the pro-fibrogenic Th2-specific cytokines $\mathrm{Il}-4$ and $\mathrm{Il}-13$ were not regulated or undetectable in AL mice (Supplementary Table S3). Fibrogenic Pdgf-B and $P d g f-D$ were also increased in both groups of WT and AL mice on WD while fibrosis-associated indian hedgehog transcription was induced in WT mice on WD only (Supplementary Table S3).

Mmp-9, Mmp-13, and their specific inhibitor Timp-1, key enzymes in liver ECM homeostasis, were significantly increased on transcriptional level in WT and AL mice on WD but not in AL mice on RD (Supplementary Figure S4a). Interestingly, MMP-9 protein activity was significantly increased in AL mice on WD compared with WT controls, as assessed by gelatin zymography (Supplementary Figure S4b).

Immunohistologically, $\mathrm{CD} 45^{+}$leukocytes within inflammatory cell aggregates were identified as the major source of MMP-9 in AL mice on WD (Supplementary Figure S4c). $\mathrm{CD}_{4}{ }^{+}$leukocytes also expressed MMP-13. However, MMP-13-producing leukocytes were not clustered together (as seen for MMP-9) but were distributed as single cells throughout the hepatic parenchyma (Supplementary Figure S4d).

\section{Assessment of Tumor in AL Mice on WD}

Throughout the whole observation period, hepatic tumors uniquely developed in AL mice on $\mathrm{WD}$, with a tumor incidence of $30 \%$ and a total number of 9 tumors. Tumor volume ranged from 10.5 to $783.4 \mathrm{~mm}^{3}$. Median tumor volume was $92.8 \mathrm{~mm}^{3}$, mean volume accounted for $207.8 \mathrm{~mm}^{3}$ with a s.d. of $272.5 \mathrm{~mm}^{3}$ and s.e. of $90.8 \mathrm{~mm}^{3}$. In contrast, no tumors developed in AL mice on RD or WT mice on any diet. Histologically, tumors were characterized by a clear and defined demarcation to surrounding healthy tissue (Figure 4a), remarkable cellular proliferation (Figures $4 \mathrm{~b}$ and c), lack of apoptosis (indicated by lack of nuclear cleaved caspase 3 staining in tumor parenchyma, data not shown), lack of fibrillar collagen and type IV collagen inside of the tumor parenchyma (Figures $4 \mathrm{~d}$ and e), and the presence of nuclear atypia (Figure 4f) and enlarged nuclei with high N/C ratio, $>2$ cell-thick plates/cords.

As a further step to assess tumor development in AL mice on WD, vascular imaging was performed from a total of 54 cubic volumes of interest (36 samples of healthy tissue, 19 samples of tumor tissue). Quantitative micro-CT imaging showed an alteration of tumor vascularization with a significantly decreased vascular volume fraction $(2.1 \% \pm 0.14$ vs $3.59 \% \pm 0.27 ; P<0.001)$ in tumor nodules compared with non-neoplastic liver tissue. The number of vessels/volume (vascular separation) was constant (data not shown) accompanied by a left shift toward smaller vessel diameters (Figures $5 \mathrm{~b}$ and $\mathrm{c}$ ), indicating tumor neovascularization.

\section{Signalling Pathways might Trigger Hepatic Tumorigenesis}

$\mathrm{I} \kappa \mathrm{B}$ protein level as well as AKT phosphorylation was markedly reduced in AL mice on WD and in neoplastic tissue (Figure 6a). Nuclear NF- $\kappa$ B translocation was observed in periportal hepatocytes of AL mice on WD but not in WT controls (Figure 6b). In contrast, immunostaining revealed the appearance of small non-parenchymal cells with strong NF- $\kappa \mathrm{B}$ accumulation in cytoplasm in WT mice on WD (Figure 6b). Phosphorylation of STAT3 and JNK was clearly induced in $\mathrm{WT}$ and $\mathrm{AL}$ mice on WD and tumor compared with WT mice on RD (Figure 6a). Interestingly phoshoSTAT3 showed the strongest signal in AL mice on RD.

\section{DISCUSSION}

NAFLD is considered to be the hepatic manifestation of the metabolic syndrome which thereby links NAFLD to the development and progression of cardiovascular disease. ${ }^{20}$ Increasing evidence of different clinical trials shows that NAFLD is a risk factor for cardiovascular disease. ${ }^{21}$ To gain insights into the molecular events underlying the association between NAFLD and cardiovascular disease, it is essential to establish and analyze murine models capable of portraying and translating the human pathophysiology.

Within the present study, we demonstrate that genetically predisposed AL mice on a cholesterol- and fat-enriched diet develop both a hepatic disorder closely resembling human NAFLD and NAFLD-associated tumorigenesis without additional carcinogen exposure. In particular, the phenotype observed in AL mice on WD distinguishes from that seen in WT mice on the same diet by pronounced inflammation, more profound metabolic changes like hyperlipidemia, a different pattern and distribution of hepatic fibrosis, and finally by spontaneous development of tumors. Thus AL mice on WD represent an established atherosclerosis mouse model that displays the most important hallmark characteristics of human NAFLD pathology.

As certain ApoE and Ldlr polymorphisms have been shown to be strongly associated with NASH risk and also with cholesterol homeostasis, we hypothesized that genetic predisposition, altered lipid metabolism, and dietary factors 
a

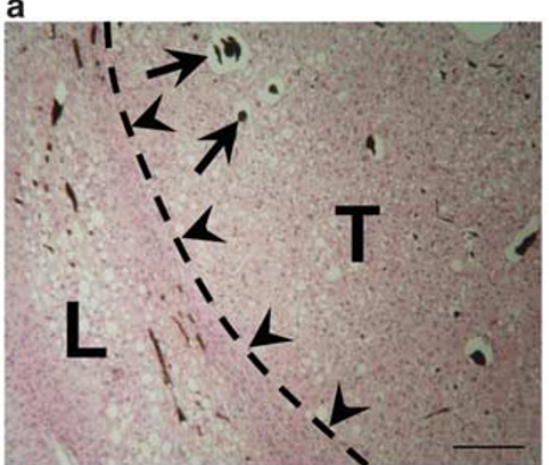

d

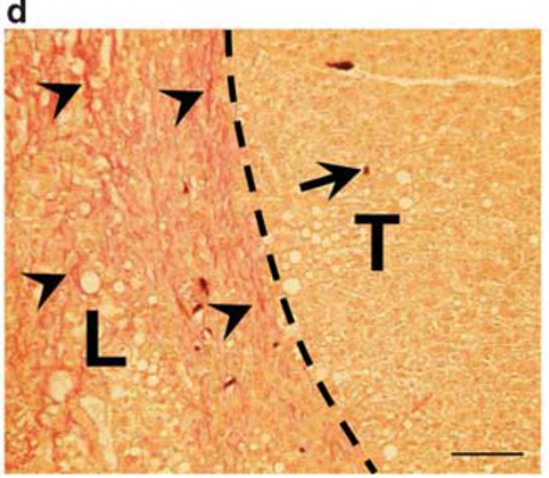

b

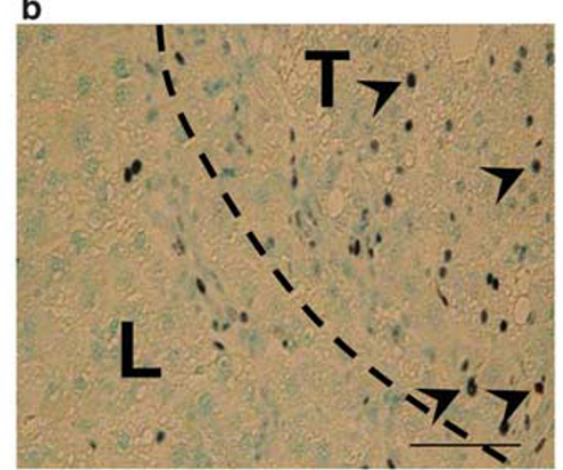

e

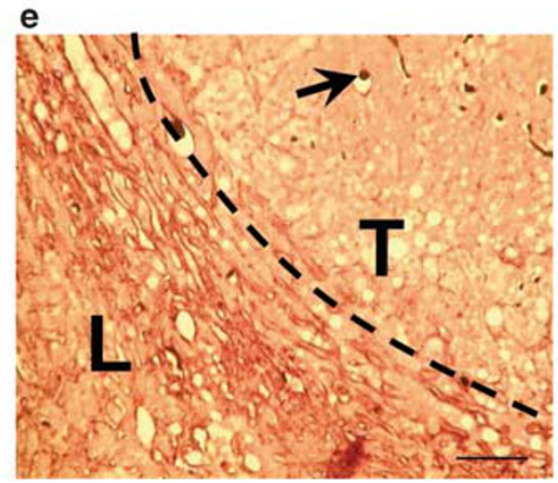

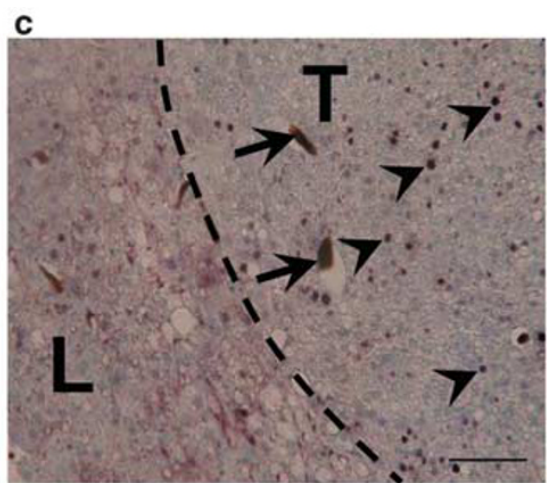

f

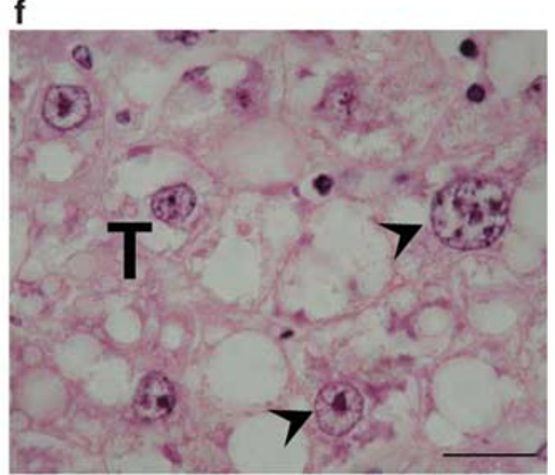

Figure 4 Histological characterization of tumors in AL mice on WD. Tumor-bearing AL mice on WD were subjected to histological analysis at 39 weeks of age. (a) A defined tumor border was visualized by H\&E staining (arrowheads). (b and c) Immunohistochemical MCM2 and PCNA stainings (arrowheads) demonstrated pronounced proliferation in the tumor (T) in comparison to surrounding liver parenchyma (L). (d) Sirius Red staining demonstrated that tumor parenchyma (T) is free from fibrillar collagen (arrowheads depict fibrillar collagen in tumor border and liver parenchyma). (e) Type IV collagen was stained on paraffin-embedded sections: type IV collagen is not expressed inside the tumor (arrowheads depict type IV collagen along with sinusoids in liver parenchyma). (f) High magnifications of neoplastic hepatocytes inside tumor lesions demonstrated nuclear atypia as indicated by arrowheads (H\&E staining). $\mathrm{L}=$ liver parenchyma, $\mathrm{T}=$ tumor parenchyma, black arrows indicate remnants of contrast medium for micro-CT analysis. Representative micrographs are shown (magnification $\times 50$ in a-e and $\times 1000$ in $\mathbf{f}$, scale bar $200 \mu \mathrm{m}$ in a-e and $20 \mu \mathrm{m}$ in $\mathbf{f}$ ).

are crucial for liver disease development. ${ }^{22,23}$ LDLR or ApoE single-knockout mice developed some of the NAFLD characteristics ${ }^{24-26}$ but generally failed to broadly reflect the whole spectrum of NAFLD key features. As such, ApoE ${ }^{-1-}$ mice on a fat- and cholesterol-enriched diet developed hepatic inflammation but only mild steatosis. Vice versa, feeding LDLR $^{-1-}$ mice with a WD led to a significant hepatic steatosis and elevated plasma triglycerides but only mild inflammation compared with $\mathrm{ApoE}^{-1-}$ mice.

Recently, the effects of a WD on atherogenesis were analyzed in AL mice. ${ }^{27}$ Although showing increased hepatic lipid accumulation in these mice, the study was not focussed on liver disease. In addition, the observation period of 12 weeks was rather short for the analysis of hepatic fibrosis and carcinogenesis as long-term sequelae of NAFLD.

To mimic the human situation of nutritional-induced NAFLD, including hyperlipidemia, we analyzed the effects of long-term nutrition of AL mice with a high-fat and highcholesterol WD within the current study. Considering the normal mouse lifespan of approximately 2.5 years, a 35 -week lasting WD is comparable to approximately 15-20 years of overnutrition in humans. Given these considerations, the treatment of AL mice with a fat- and cholesterol-enriched WD represents a relatively physiological model for the development of obesity- and dyslipidemia-based steatosis. Within our study, we demonstrate that AL mice exhibit elevated serum triglyceride and cholesterol levels and an induction of key enzymes of lipid metabolism and homeostasis. Although these elevations were also observed in $\mathrm{AL}$ mice on $\mathrm{RD}$, they were clearly pronounced in AL mice on $\mathrm{WD}$, especially when compared with WT mice on WD.

The induction of PPAR $\alpha$ is of particular interest, because PPAR $\alpha$ has been shown to have a role in both regulation of hepatic steatosis and hepatic inflammation. ${ }^{28}$ Further, in light of the increased proteins levels of FASN in AL mice on WD, increased steatosis might very well be ascribed to enhanced hepatic biosynthesis of triglycerides. This observation is consistent with findings from other studies, which were able to demonstrate the induction of triglyceride synthesis in the liver of diet-induced obesity and NAFLD. ${ }^{29}$ Therefore our data provide evidence that AL mice develop a metabolic syndrome characterized by dyslipidemia, an observation, which is of particular relevance as dyslipidemia has been shown to be a risk factor for NAFLD. Furthermore, 

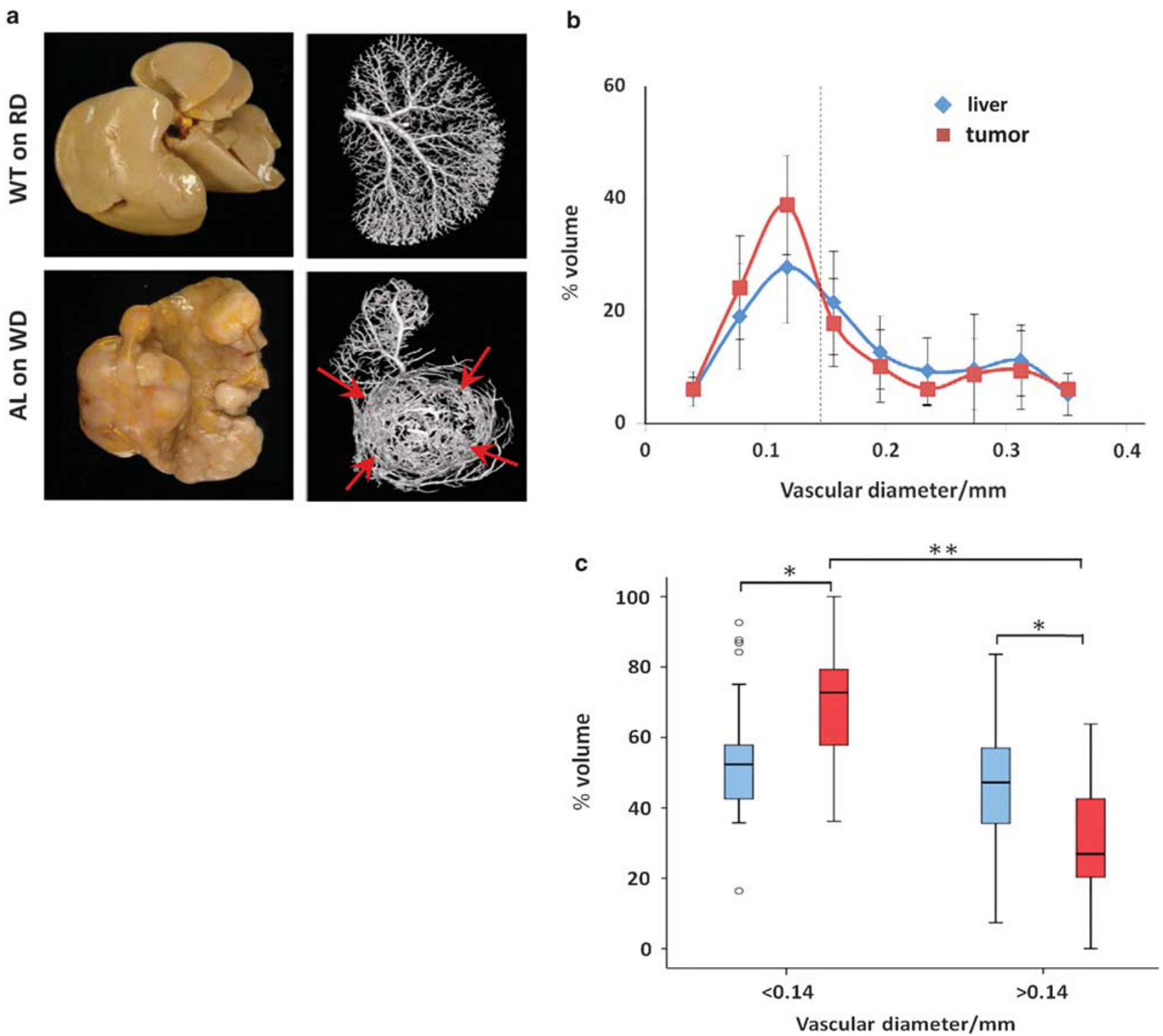

Figure 5 Altered vascularization in NASH-related tumor tissue compared with non-neoplastic tissue. (a) Macroscopic photograph of microfil perfused hepatic vasculature in a cirrhotic liver with tumor nodes compared with a liver without cirrhosis or tumor manifestation. Three-dimensional volume rendering technique (3D VRT) reformatted from Micro-CT data sets displays the differences in vascularization density in tumor nodes compared with non-neoplastic liver tissue without cirrhosis or tumor manifestation. The red arrow depicts the area of increased tumor-associated vessel clumping. (b) Quantitative analysis of vessel thickness distribution shows a left shift, indicating an increase of small vessels in tumor tissue (red curve). At the diameter of $140 \mu \mathrm{m}$ vessel thickness (vertical black line), the situation reverses: the percentage of vessels $>140 \mu \mathrm{m}$ is higher in non-neoplastic tissue (blue curve) compared with tumor tissue (red curve). (c) No significant differences of vessel diameter distribution at the cutoff level of $140 \mu \mathrm{m}$ were observed in liver tissue (blue bars). Significant differences of diameters were detected for microvessels in tumor tissue (red bars, $P<0.001)$, as well as microvessels larger and smaller than $140 \mu \mathrm{m}$, comparing the percentage of vessels smaller in tumors and non-neoplastic liver tissue $(P<0.05)$.

hyperlipidemia has been reported to be associated with hepatocellular cancer. ${ }^{30}$ In addition, even the history of hyperlipidemia has been identified to increase the risk of hepatocellular cancer of other etiology. ${ }^{31}$ Clinical evidence and the current observation of cancer development in hyperlipidemic AL mice on WD suggested that hyperlipidemia might be one important extra hit that contributes to carcinogenesis in NAFLD.
Progression of NAFLD is typically accompanied by hepatic inflammation. ${ }^{32}$ However, some of the previously described mouse NAFLD models were not commonly associated with inflammation, (eg, KK-Ay/a mice) or even developed only mild forms of inflammation, eg, ob/ob mice). In accordance with previous reports, our results indicate that WD in WT and AL mice leads to increased hepatic recruitment of inflammatory cells, increased expression of 

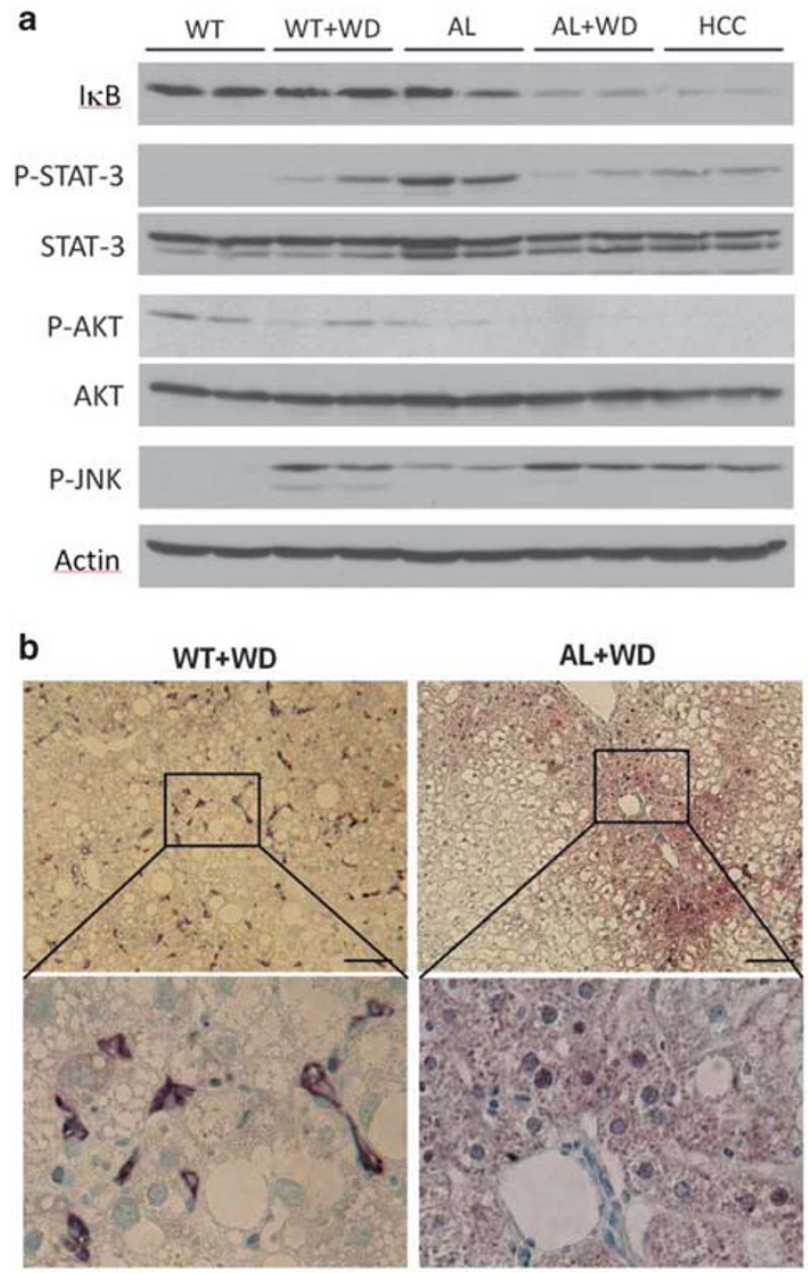

Figure 6 Concomitant signaling pattern indicating liver damage and compensatory liver regeneration. (a) Western blotting analysis revealed reduced $I \kappa B$ and P-AKT in AL mice on WD, whereas JNK signaling was induced in both WT and AL mice on WD. STAT3 is activated in WT mice by WD but reduced in AL mice on WD. Nevertheless AL mice on WD still bear more STAT3 activation than WT mice on RD. (b) Nuclear translocation of NF- $\kappa$ B was demonstrated by immunohistochemistry in AL mice on WD but not in WT mice on WD. Magnification $\times 100$ and $\times 400$. Scale bar $100 \mu \mathrm{m}$.

pro-inflammatory cytokines, and enhanced MMP and TIMP-1 expression. Importantly, leukocyte infiltration and the quantity of inflammatory foci were most prominent and pronounced in $\mathrm{AL}$ mice on $\mathrm{WD}$, indicating that the combination of genetic susceptibility and nutrition is a potent mediator of hepatic inflammation. Consistent with this finding, $\mathrm{F} 4 / 80^{+}$macrophages, myeloperoxidase ${ }^{+}$ neutrophil granulocytes, and $\mathrm{CD} 3^{+}{ }^{-}$-cells appear in cell nests with higher number in WD-fed mice and especially pronounced in AL mice on WD in comparison to WT mice on WD.

We observed quantitatively enhanced collagen expression and a NASH typical fibrosis pattern in WT and AL mice on WD within the current study. In contrast, most of the previously described mouse NASH models were not commonly associated with fibrogenesis, (eg, ob/ob, Mat1A ${ }^{-1-}$, KK-Ay/a, fructose sirup + HFD). Fibrogenesis in AL mice after WD was characterized by the appearance of $\alpha$-SMA ${ }^{+}$ myofibroblasts, which were co-localized with type-I collagen, representing at least one source for fibrotic matrix deposition. We provide further evidence that portal fibroblasts at least partly contribute to the myofibroblast population and to matrix production, and this cell type was mainly identified in AL mice on WD. This again corroborates that the combination of genetic predisposition and nutrition leads to the most profound NAFLD phenotype. ${ }^{33}$

Importantly, we observed tumors in three animals in $\mathrm{AL}$ mice on WD within the current study. During the past 8 years in which we were working with $\mathrm{AL}$ mice on $\mathrm{RD}$, development of hepatic tumors had never been observed, thereby indicating that the occurrence of hepatic malignancy is indeed uniquely limited to AL mice on WD. So far, the development of HCC-like tumor has not been reported in lipoprotein and lipoprotein receptor-defect animal models on a $\mathrm{C} 57 \mathrm{Bl} / 6$ background. Further, the dietary-induced onset of NAFLD-associated tumors in AL mice on WD in our study is considerably faster than that reported previously in related mouse models. ${ }^{34}$

ROS accumulation promotes cell death through various mechanisms, including prolonged JNK activation. ${ }^{35}$ In the current study, both ROS accumulation and prolonged JNK activation, in combination with enhanced hepatocellular injury, might drive tumorigenesis. This observation is consistent with a loss of activation of the AKT pathway, which has been identified as a key pathway for metabolism, cell growth, and cell survival. ${ }^{36}$ Nevertheless, the PI3K/AKT pathways have been discussed to be the essential link between oxidative stress and inflammation in NASH. ${ }^{36}$ Thus it is rather speculative if loss of AKT activation in AL mice on WD might be ascribed to the late phase of NAFLD with beginning carcinogenesis.

On the other hand, a marked loss of hepatic $\mathrm{I} \kappa \mathrm{B}$ protein and also nuclear translocation of NF- $\kappa \mathrm{B}$ in periportal hepatocytes of AL mice on WD clearly indicated compensatory liver regeneration. NF- $\kappa \mathrm{B}$ has a wide range of functions in different cellular compartments influencing the survival of hepatocytes, Kupffer cells, and survival and activation in HSC. ${ }^{11}$ Aberrant sustained activation of NF- $\kappa \mathrm{B}$ has been associated with chronic inflammation-derived liver cancer due to its role as a potent positive cell cycle regulator and safeguard from apoptosis. ${ }^{12}$ Therefore we speculate that NF- $\kappa \mathrm{B}$ might confer survival benefits on transforming hepatocytes in AL mice on WD. STAT3, which is also involved in cell survival and proliferation and has been linked to NF- $\kappa \mathrm{B}$ pathway with regard to HCC development, was also activated in $\mathrm{AL}$ and WT mice on WT. ${ }^{10}$ Taken together, these results demonstrate that chronic inflammatory conditions in steatotic livers of AL mice on WD went along with the induction of pathways linked to liver damage on the 
one hand and compensatory liver regeneration on the other hand. Opposing signaling and subsequent hepatocellular reactions might complement optimal conditions for tumor development in AL mice on WD. Nevertheless, NF- $\kappa \mathrm{B}$ activation and the loss of AKT activation in combination with increased inflammation in AL mice on WD is the major difference to WT mice on WD and thus might be involved in carcinogenesis.

Tumor imaging in small rodents is an emerging technique. Beyond the initial use of micro-CT for evaluation of highcontrast objects such as bone, ${ }^{37}$ the spectrum of micro-CT applications has expanded recently, presenting micro-CT imaging as a valuable tool in tumor imaging nowadays. ${ }^{38}$ Herein, we demonstrate that micro-CT is capable of visualizing tumor vascularization and of distinguishing tumor tissue from non-neoplastic liver tissue. In combination with the three-dimensional micro-CT data, the architecture of vascular trees and their distribution pattern were visualized and quantified. Our data provide evidence that the vascular pattern is structurally different in neoplastic liver tissue compared with non-neoplastic hepatic tissue. Thus this report is the first to describe micro-CT in combination with advanced contrast enhancement as a novel and promising technique for ultrastructural and threedimensional ex vivo imaging of hepatic tumor tissue.

\section{Limitations}

Apart from the presented observations of tumorigenesis in WD-fed AL mice, Chi-square analyis did not reveal significance for the development of tumors in AL mice. This may be explained by the limited number of animals and raises the need of further studies including larger animal cohorts.

In conclusion, we characterized hepatic pathogenesis in a murine model for the development of atherosclerosis. As identified within this study, AL mice on WD display hepatic steatosis, dyslipidemia, infiltration of inflammatory cells, fibrosis, and tumorigenesis. Signaling pathways of liver cell damage and compensatory liver regeneration in combination with enhanced inflammation appear to be crucial for the spontaneous development of tumors in AL mice on WD. This model might be a valuable tool to study metabolic and immunological mechanisms linking NAFLD to atherosclerosis-associated complications.

Supplementary Information accompanies the paper on the Laboratory Investigation website (http://www.laboratoryinvestigation.org)

\section{ACKNOWLEDGMENTS}

The authors are grateful to Gunhild Martels, Annette Tschuschner, Jennifer Roth, and Boris Wilde for excellent technical assistance. This work was supported by grants from the Deutsche Forschungsgemeinschaft (RO 957/ 8-1), by the faculty of medicine, the von Behring Röntgen Foundation, and by a Research Grant from the University Hospital Giessen and Marburg (UKGM 7/2014 Gl).

\section{DISCLOSURE/CONFLICT OF INTEREST}

The authors declare no conflict of interest.

1. Ahmed MH, Barakat S, Almobarak AO. Nonalcoholic fatty liver disease and cardiovascular disease: has the time come for cardiologists to be hepatologists? J Obes 2012;2012:483135.

2. Armstrong MJ, Houlihan DD, Bentham L, et al. Presence and severity of non-alcoholic fatty liver disease in a large prospective primary care cohort. J Hepatol 2012;56:234-240.

3. Williams CD, Stengel J, Asike MI, et al. Prevalence of nonalcoholic fatty liver disease and nonalcoholic steatohepatitis among a largely middleaged population utilizing ultrasound and liver biopsy: a prospective study. Gastroenterology 2011;140:124-131.

4. Argo CK, Caldwell SH. Epidemiology and natural history of nonalcoholic steatohepatitis. Clin Liver Dis 2009;13:511-531.

5. Hashimoto E, Tokushige K. Prevalence, gender, ethnic variations, and prognosis of NASH. J Gastroenterol 2011;46(Suppl 1):63-69.

6. Day CP, James OF. Steatohepatitis: a tale of two "hits"? Gastroenterology 1998;114:842-845.

7. Wouters K, van Gorp PJ, Bieghs V, et al. Dietary cholesterol, rather than liver steatosis, leads to hepatic inflammation in hyperlipidemic mouse models of nonalcoholic steatohepatitis. Hepatology 2008;48: 474-486.

8. Hijona E, Hijona L, Arenas Jl, et al. Inflammatory mediators of hepatic steatosis. Mediators Inflamm 2010;2010:837419.

9. Langheinrich AC, Michniewicz A, Bohle RM, et al. Vasa vasorum neovascularization and lesion distribution among different vascular beds in ApoE-/-/LDL-/- double knockout mice. Atherosclerosis 2007;191:73-81.

10. He G, Karin M. NF-kappaB and STAT3 - key players in liver inflammation and cancer. Cell Res 2011;21:159-168.

11. Luedde T, Schwabe RF. NF-kappaB in the liver-linking injury, fibrosis and hepatocellular carcinoma. Nat Rev Gastroenterol Hepatol 2011;8:108-118.

12. Pikarsky E, Ben-Neriah Y. NF-kappaB inhibition: a double-edged sword in cancer? Eur J Cancer 2006:42:779-784.

13. Roderfeld $M$, Rath $T$, Voswinckel $R$, et al. Bone marrow transplantation demonstrates medullar origin of CD34 + fibrocytes and ameliorates hepatic fibrosis in Abcb4-/- mice. Hepatology 2010;51:267-276.

14. Batts KP, Ludwig J. Chronic hepatitis. An update on terminology and reporting. Am J Surg Pathol 1995;19:1409-1417.

15. Ishak K, Baptista A, Bianchi L, et al. Histological grading and staging of chronic hepatitis. J Hepatol 1995;22:696-699.

16. Levene AP, Kudo H, Armstrong MJ, et al. Quantifying hepatic steatosis - more than meets the eye. Histopathology 2012;60:971-981.

17. Maeda S, Kamata H, Luo JL, et al. IKKbeta couples hepatocyte death to cytokine-driven compensatory proliferation that promotes chemical hepatocarcinogenesis. Cell 2005;121:977-990.

18. Jamall IS, Finelli VN, Que Hee SS. A simple method to determine nanogram levels of 4-hydroxyproline in biological tissues. Anal Biochem 1981;112:70-75.

19. Herron GS, Banda MJ, Clark EJ, et al. Secretion of metalloproteinases by stimulated capillary endothelial cells. II. Expression of collagenase and stromelysin activities is regulated by endogenous inhibitors. J Biol Chem 1986;261:2814-2818.

20. Pacana $\mathrm{T}$, Fuchs $\mathrm{M}$. The cardiovascular link to nonalcoholic fatty liver disease: a critical analysis. Clin Liver Dis 2012;16:599-613.

21. Scorletti E, Calder PC, Byrne CD. Non-alcoholic fatty liver disease and cardiovascular risk: metabolic aspects and novel treatments. Endocrine 2011;40:332-343.

22. Ling IF, Estus S. Role of SFRS13A in low-density lipoprotein receptor splicing. Hum Mutat 2010;31:702-709.

23. Sazci A, Akpinar G, Aygun C, et al. Association of apolipoprotein E polymorphisms in patients with non-alcoholic steatohepatitis. Dig Dis Sci 2008;53:3218-3224.

24. Bieghs V, Van Gorp PJ, Wouters K, et al. LDL receptor knock-out mice are a physiological model particularly vulnerable to study the onset of inflammation in non-alcoholic fatty liver disease. PLoS One 2012; 7:e30668.

25. Gupte AA, Liu JZ, Ren Y, et al. Rosiglitazone attenuates age- and diet-associated nonalcoholic steatohepatitis in male low-density lipoprotein receptor knockout mice. Hepatology 2010;52:2001-2011. 
26. Lalloyer F, Wouters K, Baron M, et al. Peroxisome proliferator-activated receptor-alpha gene level differently affects lipid metabolism and inflammation in apolipoprotein E2 knock-in mice. Arterioscler Thromb Vasc Biol 2011;31:1573-1579.

27. Zou $Y$, Du H, Yin $M$, et al. Effects of high dietary fat and cholesterol on expression of PPAR alpha, LXR alpha, and their responsive genes in the liver of apoE and LDLR double deficient mice. Mol Cell Biochem 2009;323:195-205.

28. Tanaka N, Moriya K, Kiyosawa $\mathrm{K}$, et al. PPARalpha activation is essential for HCV core protein-induced hepatic steatosis and hepatocellular carcinoma in mice. J Clin Invest 2008;118:683-694.

29. Dorn C, Riener MO, Kirovski G, et al. Expression of fatty acid synthase in nonalcoholic fatty liver disease. Int J Clin Exp Pathol 2010;3:505514.

30. Nagaoki $\mathrm{Y}$, Hyogo $\mathrm{H}$, Aikata $\mathrm{H}$, et al. Recent trend of clinical features in patients with hepatocellular carcinoma. Hepatol Res 2012;42: 368-375.

31. Cho ER, Shin A, Choi KS, et al. Factors associated with use of ultrasonography screening for hepatocellular carcinoma among hepatitis $B$ or C carriers. Cancer Epidemiol 2010;34:713-716.
32. Brunt EM. Nonalcoholic steatohepatitis: definition and pathology. Semin Liver Dis 2001;21:3-16.

33. Li Z, Dranoff JA, Chan EP, et al. Transforming growth factor-beta and substrate stiffness regulate portal fibroblast activation in culture. Hepatology 2007:46:1246-1256.

34. Hill-Baskin AE, Markiewski MM, Buchner DA, et al. Diet-induced hepatocellular carcinoma in genetically predisposed mice. Hum Mol Genet 2009;18:2975-2988.

35. Kamata $\mathrm{H}$, Honda $\mathrm{S}$, Maeda $\mathrm{S}$, et al. Reactive oxygen species promote TNFalpha-induced death and sustained JNK activation by inhibiting MAP kinase phosphatases. Cell 2005;120:649-661.

36. Matsuda S, Kobayashi M, Kitagishi Y. Roles for PI3K/AKT/PTEN pathway in cell signaling of nonalcoholic fatty liver disease. ISRN Endocrinol 2013;2013:472432

37. Elliott JC, Dover SD. Three-dimensional distribution of mineral in bone at a resolution of 15 micron determined by x-ray microtomography. Metab Bone Dis Relat Res 1984;5:219-221.

38. Savai R, Langheinrich AC, Schermuly RT, et al. Evaluation of angiogenesis using micro-computed tomography in a xenograft mouse model of lung cancer. Neoplasia 2009;11:48-56. 